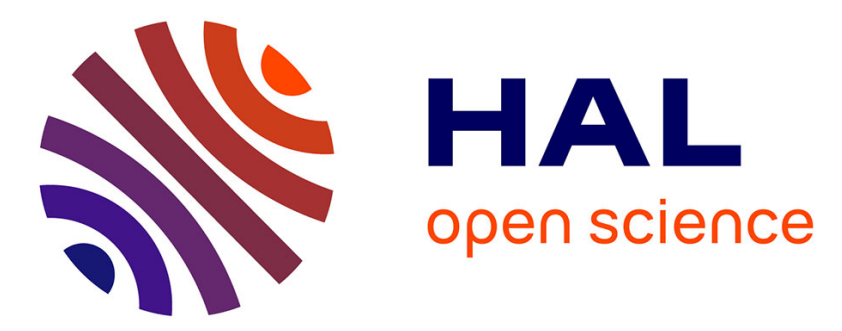

\title{
Multiple light scattering in metallic ejecta produced under intense shockwave compression
}

Jean Eloi Franzkowiak, P. Mercier, G. Prudhomme, Laurent Berthe

\section{To cite this version:}

Jean Eloi Franzkowiak, P. Mercier, G. Prudhomme, Laurent Berthe. Multiple light scattering in metallic ejecta produced under intense shockwave compression. Applied optics, 2018, 57 (11), pp.27662772. 10.1364/AO.57.002766 . hal-02163557

\section{HAL Id: hal-02163557 https://hal.science/hal-02163557}

Submitted on 24 Jun 2019

HAL is a multi-disciplinary open access archive for the deposit and dissemination of scientific research documents, whether they are published or not. The documents may come from teaching and research institutions in France or abroad, or from public or private research centers.
L'archive ouverte pluridisciplinaire HAL, est destinée au dépôt et à la diffusion de documents scientifiques de niveau recherche, publiés ou non, émanant des établissements d'enseignement et de recherche français ou étrangers, des laboratoires publics ou privés. 


\title{
Multiple light scattering in metallic ejecta produced under intense shockwave compression
}

\author{
J.-E. Franzkowiak, ${ }^{1,2, *}$ P. Mercier, ${ }^{1}$ G. Prudhomme, ${ }^{1}$ and L. Berthe ${ }^{2}$ \\ ${ }^{1}$ CEA-DAM-DIF, F-91297, Arpajon, France \\ 2PIMM UMR 8006 CNRS-Arts et Métiers ParisTech, 151 boulevard de l'Hôpital, F-75013 Paris, France \\ *Corresponding author: jean-eloi.franzkowiak@ensam.eu
}

\begin{abstract}
A roughened metallic plate, subjected to intense shock wave compression, gives rise to an expanding ejecta particle cloud. Photonic Doppler velocimetry (PDV), a fiber-based heterodyne velocimeter, is often used to track ejecta velocities in dynamic compression experiments and on nanosecond time scales. Shortly after shock breakout at the metal-vacuum interface, a particular feature observed in many experiments in the velocity spectrograms is what appear to be slow-moving ejecta, below the free-surface velocity. Using Doppler Monte Carlo simulations incorporating the transport of polarization in the ejecta, we show that this feature is likely to be explained by the multiple scattering of light, rather than by possible collisions among particles, slowing down the ejecta. As the cloud expands in a vacuum, the contribution of multiple scattering decreases due to the limited field of view of the pigtailed collimator used to probe the ejecta, showing that the whole geometry of the system must be taken into account in the calculations to interpret and predict PDV measurements. (c) 2018 Optical Society of America
\end{abstract}

\section{INTRODUCTION}

As a shockwave reaches the free surface of a roughened metal, high-speed ejecta particles are produced from a limiting case (Atwood number $A \simeq-1$ ) of the Richtmyer-Meshkov instability phenomena [1] and freely expand in a vacuum, constituting an expanding cloud of droplets. To measure the areal mass of ejecta, Asay foil [2], x-ray [3,4], and piezoeletric pins [5] have been commonly used in dynamic compression experiments. Photonic Doppler velocimetry (PDV), a fiber-based heterodyne velocimeter [6-8], is employed to measure particle velocities in their direction of expansion and is a promising diagnostic for a non-invasive characterization of ejecta particles. This effort investigates the multiple scattering of light in the ejecta and its influence on time-velocity spectrograms measured by PDV. What appear to be slow-moving ejecta detected by PDV shortly after breakout (below the free-surface) are explained by collisions among ejecta particles in Ref. [9]. As already presented in Ref. [10] in the case of a micro-spall particle cloud (particle sizes in the range of $10-100 \mu \mathrm{m}$ and velocity gradients in the range of $300 \mathrm{~m} / \mathrm{s}$ ), we suggest an explanation to this observation in the case of a micro-jetting particle cloud (particle sizes in the range of $0.5-10 \mu \mathrm{m}$ and velocity gradients in the range of $1000-2000 \mathrm{~m} / \mathrm{s}$ ), based on the multiple scattering of light in the ejecta. The solution is obtained using a Doppler Monte Carlo (MC) approach, taking into account the transport of polarization in the ejecta cloud. Furthermore, we link the contribution of multiple scattering to the geometry of the PDV pigtailed collimator, justifying why this particular signature decreases with time, and showing that the whole geometry of the system must be taken into account to interpret precisely PDV measurements in metallic ejecta experiments.

\section{METHODS}

In a PDV setup, a collimated and coherent light beam at $\lambda=1.55 \mu \mathrm{m}$ and frequency $\omega_{0}$ is used to illuminate an expanding metallic particle cloud. Doppler-shifted scattered fields are collected in the pigtailed collimator in the backscattering direction and mixed with a reference beam (local oscillator) with frequency $\omega_{\mathrm{LO}}=\omega_{0}$ and intensity $P_{\mathrm{LO}}$, using a heterodyne configuration, to generate an intensity modulation $I(t)$ in the photodetector plane. The square modulus of the short-term Fourier transform (STFT) calculated on $I(t)$ is the optical power spectral density (PSD), so-called time-velocity PDV spectrograms in the shock community. To emphasize how the multiple scattering of light impacts PDV results, we perform Doppler MC simulations. Doppler MC is a powerful tool, often employed in tissue optics and laser Doppler flowmetry [11-13], to simulate complex systems. The Doppler spectrum, originating from the interaction of a coherent light 
beam with moving scatterers, can be recovered using such statistical methods. We employed this method in our study due to the high complexity of the ejecta cloud: the polydispersity must be taken into account, as well as the spatial evolution of volume particle densities, from the head of the cloud to the location of the free surface.

\section{A. Description of the Cloud}

A particle cloud evolving freely in vacuum along $z$ is defined by its particle size and velocity distributions (resp., $f_{d}$ and $f_{v}$ ), between diameters $d_{\min }$ and $d_{\max }$, and between free-surface velocity $V_{\mathrm{fs}}$ and $V_{\max } \cdot f_{v}(V) d V$ is the approximate number of particles between velocities $V$ and $V+d V$, and $f_{d}\left(d_{p}\right) d d_{p}$ the number of particles between diameters $d_{p}$ and $d_{p}+d d_{p}$. The integrated areal mass-velocity function of ejecta $M(V)$ $\left(\mathrm{kg} / \mathrm{m}^{2}\right)$ between $V$ and $V_{\text {max }}$ is defined by

$$
M(V) \simeq \frac{\rho_{p}\left\langle V_{p}\right\rangle}{S} \int_{V}^{V_{\max }} f_{v}(V) \mathrm{d} V,
$$

with $\rho_{p}\left(\mathrm{~kg} / \mathrm{m}^{3}\right)$ the material density and $S\left(\mathrm{~m}^{2}\right)$ the surface of ejection. The total areal mass of ejecta is $M_{s}=M\left(V_{\mathrm{fs}}\right)$. $\left\langle V_{p}(V)\right\rangle\left(\mathrm{m}^{3}\right)$ is an average particle volume determined from the normalized size distribution $f_{d}$ :

$$
\left\langle d_{p}\right\rangle^{3}=\int_{d_{\min }}^{d_{\max }} d_{p}^{3} f_{d}\left(d_{p}\right) \mathrm{d} d_{p}=\frac{6}{\pi}\left\langle V_{p}\right\rangle .
$$

We have assumed that particle sizes and velocities are uncorrelated. This may not be exactly true, and a dependence on velocity may be introduced to account for correlations between particle sizes and velocities, $d_{\max }$ increasing with decreasing velocities [14]. However, this dependence is very small, and we assume uncorrelated sizes and velocities will not change the results presented in this paper.

A pigtailed collimator (lens diameter $\Phi_{p}$ ) is used to illuminate ejecta particles in their direction of propagation $z$. Scattered fields are collected in the same aperture in the backscattering direction. The solid angle $\Omega$ within which photons are collected is limited by the fiber core diameter $\Phi_{c}$ and the focal length $f^{\prime}$ of the probe through the relationship $\Omega=\pi \Phi_{c}^{2} /\left(4 f^{\prime 2}\right)=\pi \tan \alpha^{2}$. Below a critical distance $d_{c}=\Phi_{p} /(2 \tan \alpha)$, the optical coupling efficiency of the scattered fields in the system is constant. Shortly after breakout, when liquid metal sheets have broken up into micrometer-sized particles, the cloud of length $L$ is discretized into $N$ slabs of width $\delta z=L / N$, to each of which is associated a velocity (in decreasing order) $V_{i} \in\left[V_{\max }, V_{\mathrm{fs}}\right]$ and a scattering mean free path $1 / \mu_{i}(\mathrm{~m})$ defined, $\forall i \in[1, N]$ and using the independent scattering approximation, by

$$
\mu_{i}=\frac{3\left\langle Q_{\mathrm{ext}}\right\rangle \delta M\left(V_{i}\right)}{2 \rho_{p} \delta z\left\langle d_{p}\right\rangle},
$$

with $\delta M\left(V_{i}\right)=M\left(V_{i+1}\right)-M\left(V_{i}\right)\left(\mathrm{kg} / \mathrm{m}^{2}\right)$ and

$$
\left\langle Q_{\text {ext }}\right\rangle=\int_{d_{\max }}^{d_{\min }} f_{d}\left(d_{p}\right) Q_{\text {ext }}\left(d_{p}\right) \mathrm{d} d_{p},
$$

the average extinction efficiency determined using Mie theory of light scattering (spherical particles). $Q_{\text {ext }}\left(d_{p}\right)$ is the extinction efficiency for a single spherical particle of diameter $d_{p}$. Equation (3) is valid if particle volume fractions do not exceed $\sim 1 \%$, otherwise correlations between scatterers will imply a nonlinear dependence of $\mu_{i}$ with particle densities [15].

\section{B. Doppler Monte Carlo Simulations}

In the preceding section, the optical properties of the ejecta particle cloud have been defined and MC simulations can be performed to simulate the interaction. A photon is launched with initial weight $W_{1}=1$, direction $[0 ; 0 ; 1]=$ $\left[u_{1, x} ; u_{1, y} ; u_{1, z}\right]=\boldsymbol{u}_{1}$, position $\left[x_{1} ; y_{1} ; 0\right]$, and null Doppler shift $V_{d}(\mathrm{~m} / \mathrm{s})$ (Fig. 1), $x_{1}$ and $y_{1}$ being sampled using a Gaussian distribution of waist $\omega$. A pseudo-random number $\eta$ between 0 and 1 is sampled and $j$ initialized to 1 . As $-\log \eta$ remains smaller than $\delta z \sum_{i=1}^{j} \mu_{i}, j$ is incremented by one and the distance $\ell_{s}$ traveled before a first scattering by $\delta z$. When the inequality is violated,

$$
\left.\ell_{s}=(j-1) \delta z-\frac{1}{\mu_{j}} \log \eta+\delta z \sum_{i=1}^{j-1} \mu_{i}\right),
$$

a scattering occurs on a particle of diameter $d_{p, 1}$ and velocity $\mathbf{V}_{1}=-V_{1} \mathbf{u}_{z}$. Assuming a power-law dependence [16] for particle sizes $f_{d} \propto d_{p}^{-\alpha_{c}}, d_{p, 1}$ is directly sampled using the inverse cumulative distribution function and the random deviate $\eta$ :

$$
d_{p, 1}=\left[\left(d_{\max }^{1-\alpha_{c}}(V)-d_{\min }^{1-\alpha_{c}}\right) \eta+d_{\min }^{1-\alpha_{c}}\right]^{\frac{1}{1-\alpha_{c}}} .
$$

To get the direction of the photon $\mathbf{u}_{2}$ after a first scattering, the scattering properties of the particle must be defined. The single scattering matrix is expressed as

$$
\mathbf{M}(\theta)=\left(\begin{array}{cccc}
a(\theta) & b(\theta) & 0 & 0 \\
b(\theta) & a(\theta) & 0 & 0 \\
0 & 0 & d(\theta) & -e(\theta) \\
0 & 0 & e(\theta) & d(\theta)
\end{array}\right),
$$

with

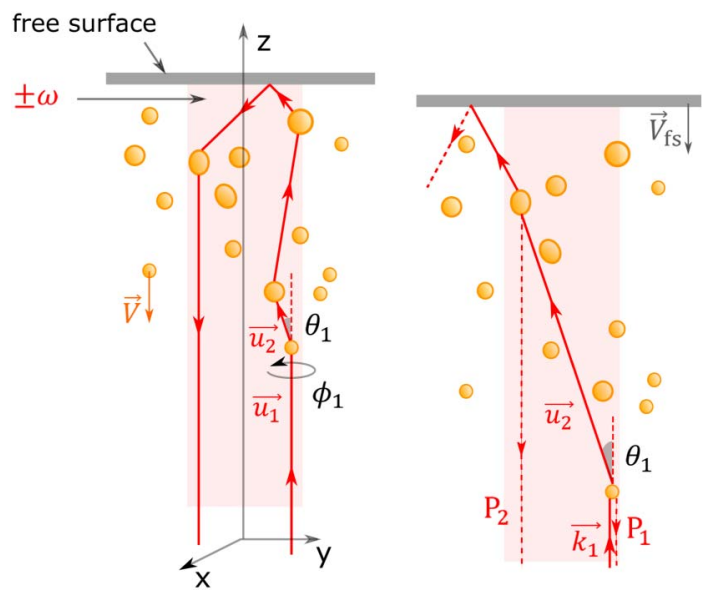

Fig. 1. Photon path in the ejecta at two different instants. Velocities are colinear to the $z$ axis. At late time (right), the impact of multiple scattering on the measurement is reduced due to the limited FOV of the probe lens. 


$$
\begin{aligned}
a(\theta) & =\frac{1}{2}\left(\left|S_{1}\right|^{2}+\left|S_{2}\right|^{2}\right), \\
b(\theta) & =\frac{1}{2}\left(\left|S_{2}\right|^{2}-\left|S_{1}\right|^{2}\right), \\
d(\theta) & =\frac{1}{2}\left(S_{2} S_{1}^{*}+S_{1} S_{2}^{*}\right), \\
e(\theta) & =\frac{i}{2}\left(S_{2} S_{1}^{*}-S_{1} S_{2}^{*}\right) .
\end{aligned}
$$

$S_{1}$ and $S_{2}$ define the complex amplitude scattering matrix calculated using Mie theory. Note that matrices $\mathbf{M}(\theta)$ are calculated for particle diameters ranging from $d_{\min }$ to $d_{\max }$ before the MC simulation starts. To incorporate the transport of a polarized light beam in the ejecta cloud, we follow the Euler MC method described in Ref. [11]. For an incident Stokes vector $\mathbf{S}_{0}=\left[I_{0}, Q_{0}, U_{0}, V_{0}\right]$, the scattering phase function $p_{s}(\theta, \phi)$ is defined by

$$
p_{s}(\theta, \phi)=a(\theta) I_{0}+b(\theta)\left[Q_{0} \cos 2 \phi+U_{0} \sin 2 \phi\right] .
$$

At each photon-particle interaction site, the polar and azimuthal angles $\theta$ and $\phi$ are sampled using a rejection method [Eq. (8)]. The new direction of the photon is given by $\mathbf{u}_{2}=\mathcal{R}_{\theta_{1}} \mathcal{R}_{\phi_{1}} \mathbf{u}_{1}, \mathcal{R}_{\theta_{1}}$ being the rotation matrix of angle $\theta_{1}$ in $3 \mathrm{D}$ space around the $y$ axis and $\mathcal{R}_{\phi_{1}}$ the rotation matrix of angle $\phi_{1}$ around the $z$ axis. The weight $W_{1}$ is decreased using an implicit scheme to account for part of the radiation being absorbed by the metal: $W_{2}=W_{1}\left[1-Q_{\text {abs }}\left(d_{p, 1}\right) / Q_{\text {ext }}\left(d_{p, 1}\right)\right]$. The Doppler shift in velocity $V_{d}(\mathrm{~m} / \mathrm{s})$ is also incremented according to $V_{d}=V_{d}+0.5\left(\mathbf{u}_{2}-\mathbf{u}_{1}\right) \cdot \mathbf{V}_{1}$. The incident Stokes vector $\mathbf{S}_{0}$ is transformed into a scattered Stokes vector $S$ upon scattering. As described in Ref. [11], using the $4 \times 4$ matrix defined by

$$
\mathbf{Q}(\phi)=\left(\begin{array}{cccc}
1 & 0 & 0 & 0 \\
0 & \cos 2 \phi & \sin 2 \phi & 0 \\
0 & -\sin 2 \phi & \cos 2 \phi & 0 \\
0 & 0 & 0 & 1
\end{array}\right)
$$

we get

$$
\mathbf{S}=\mathbf{M}\left(\theta_{1}\right) \mathbf{Q}\left(\phi_{1}\right) \mathbf{S}_{0},
$$

defined in the local Euler reference frame of the photon. From this position, a new distance $\ell_{s}$ is sampled to determine the location of the next scattering event, etc. The free surface is treated as a perfect reflector moving at velocity $\mathbf{V}_{\mathrm{fs}}=-V_{\mathrm{fs}} \mathbf{u}_{z}$. To speed up the calculation, a variance reduction technique is used: as scattering events remain within the field of view (FOV) (see Fig. 1) of the PDV probe $( \pm \omega)$, we store the probability that, after each scattering, the photon is collected in the backscattering direction. The photon being scattered $n_{e}$ times in the cloud within the FOV, for $k \in\left[1, n_{e}\right]$, the probability of detection, corrected from an exponential attenuation factor, is

$$
P_{k}=\prod_{\ell=1}^{k}\left(1-\frac{Q_{\mathrm{abs}, \ell}}{Q_{\mathrm{ext}, \ell}}\right) \exp \left[-\sum_{i=1}^{j_{k}} \mu_{i} \delta z\right] p_{k}\left(\pi-\theta^{(k)}\right) .
$$

$j_{k}$ is the index of the layer in which each scattering occurs, and $p_{k}$ is the probability of backscattering for the $k$-th scattering on a particle of diameter $d_{p, k} . \theta^{(k)}$ is the angle between $-\mathbf{u}_{z}$ and $\mathbf{u}_{k}$. If the photon propagates towards the probe (decreasing $z$ ), $p_{k}\left(\pi-\theta^{(k)}\right)$ is replaced with $p_{k}\left(\theta^{(k)}\right)$ in Eq. (11). To $P_{k}$ is associated a given Doppler shift $V_{d, k}(\mathrm{~m} / \mathrm{s})$, accumulated by the photon over the scattering path (Doppler frequency $\omega_{k}$ ):

$$
V_{d, k}=\frac{\lambda}{4 \pi} \omega_{k}=-\left(\mathbf{u}_{z}+\mathbf{u}_{k}\right) \cdot \frac{\mathbf{V}_{k}}{2}+\frac{1}{2} \sum_{\ell=1}^{k-1}\left(\mathbf{u}_{\ell+1}-\mathbf{u}_{\ell}\right) \cdot \mathbf{V}_{\ell},
$$

and a given Stokes vector $\boldsymbol{S}_{k}$, calculated after $k$ scattering events. At each interaction site, we determine the backscattered Stokes vector. A last transformation, which rotates $\boldsymbol{S}_{k}$ to the meridian plane of the probe, must be computed to get the final Stokes vector $\boldsymbol{S}_{k, f}$ in the reference plane (Oxy), shown in Fig. 1. This rotation is computed using the local Euler basis (see Ref. [11]). The backscattered complex field amplitude $\mathbf{E}(t)$ can be described by a sum over all different paths $k$ followed by the photons in the ejecta:

$$
\mathbf{E}(t) \propto \sum_{k} \sqrt{P_{k}} \exp \left[i\left(\left(\omega_{0}+\omega_{k}\right) t+\phi_{k}\right)\right] \mathbf{e}_{k, f},
$$

where $\mathbf{e}_{k, f}$ is a unitary vector defining the polarization state of each backscattered photon associated to the Stokes vector $\mathbf{S}_{k f}$, $P_{k}$ is given by Eq. (11), and $\phi_{k}$ and $\omega_{k}$ are, respectively, the phase and the Doppler frequency associated to each path $\left(\phi_{k}\right.$ are uniformly distributed between $-\pi$ and $\pi$ ). The intensity of the local oscillator $P_{\mathrm{LO}}$ being much larger than the intensity scattered by the cloud, we can neglect the homodyne interference term (mutual interferences between the scattered waves), as well as the components oscillating at optical frequencies $2\left(\omega_{0}+\omega_{k}\right)$ (DC terms). The beating intensity $I(t)$ measured by the photodetector is proportional to (heterodyne interference):

$$
I(t) \propto 2 \sqrt{P_{\mathrm{LO}}} \sum_{k} \sqrt{P_{k}} \exp \left[i\left(\omega_{k} t+\phi_{k}-\phi_{\mathrm{LO}}\right)\right] \mathbf{e}_{k, f} \cdot \mathbf{e}_{\mathrm{LO}}+\text { c.c. },
$$

where "c.c." stands for "complex conjugate." $\mathbf{e}_{\mathrm{LO}}$ is the unit norm vector defining the polarization state of the local oscillator with constant phase $\phi_{\mathrm{LO}}$. Following the reasoning presented in Ref. [13] (Appendix A), we define $N$ the total number of frequency intervals in the spectrum, and we assume that all photon paths corresponding to the same frequency (number of paths $\gg 1$ ) can be merged into a single Doppler frequency component $\omega_{j}$, as done in Ref. [10]. Using the $\delta$-Kronecker notation, Eq. (14) simplifies to

$$
\begin{aligned}
I(t) \propto & 2 \sqrt{P_{\mathrm{LO}}} \sum_{j=1}^{N} \sum_{k} \sqrt{P_{k}} \exp \left[i\left(\omega_{k} t+\phi_{k}-\phi_{\mathrm{LO}}\right)\right] \\
& \times \delta\left(\omega_{k}-\omega_{j}\right) \mathbf{e}_{k, f} \cdot \mathbf{e}_{\mathrm{LO}}+\text { c.c. } \\
I(t) \propto 2 & \sqrt{P_{\mathrm{LO}}} \sum_{j=1}^{N} \sqrt{P_{j}^{\prime}} \exp \left[i\left(\omega_{j} t+\phi_{j}\right)\right]+\text { c.c.. }
\end{aligned}
$$

$P_{j}^{\prime}$ and $\phi_{j}$ (uniformly distributed between $-\pi$ and $\pi$ ) depend on time $t$. The expectation value of $P_{j}^{\prime}$ is given by

$$
\left\langle P_{j}^{\prime}\right\rangle=\sum_{k} P_{k} \mathcal{C}_{k} \delta\left(\omega_{k}-\omega_{j}\right),
$$

with $\mathcal{C}_{k} \in[0,1]$ the interference contrast between $\mathbf{e}_{k, f}$ and $\mathbf{e}_{\mathrm{LO}}$ : 


$$
\mathcal{C}_{k}=\left\|\mathbf{e}_{k, f} \cdot \mathbf{e}_{L O}\right\|^{2} .
$$

$\mathbf{e}_{k, f}$ and $\mathbf{e}_{\mathrm{LO}}$ represent two unique points on the surface of the Poincaré sphere. If $\mathbf{e}_{k}=\mathbf{e}_{\mathrm{LO}}, \mathcal{C}_{k}=1$. If there are no preferential backscattered polarizations, a polarization diversity receiver may be used to mitigate speckle fluctuations in the PDV spectrum [17]. The values of $\mathcal{C}_{k}$ for two different polarization states are uniquely determined by the distance between these two points on the surface of the Poincaré sphere. After a few scatterings, the polarization states of the backscattered photons will be randomized, and $\mathcal{C}_{k}$ will decrease to an average value of $1 / 2$.

Since all particles are moving in the cloud, a Doppler shift is calculated for each scattering event. A typical PDV probe $\left(\Phi_{c} \simeq 8 \mu \mathrm{m}, f^{\prime} \simeq 2.5 \mathrm{~mm}\right)$ has an aperture angle $\alpha \simeq 0.1^{\circ}$, and it will be assumed that only backscattered photons contribute to the Doppler spectrum ( $\cos \alpha \simeq 1$, an angle of $0.1^{\circ}$ having a negligible impact on the Doppler measurement). A photon exiting two times the FOV is terminated in the calculation. The Fourier transform of the autocorrelation of the intensity signal $I(t) \propto E E^{*}$ can be calculated to determine the PSD (see Ref. [13]). Finally, the authors show in Ref. [13] that the heterodyne Doppler spectrum at a given frequency $\omega_{j}$ is proportional to the optical energy detected in the corresponding domain. Using this result, and $n_{\mathrm{ph}}$ photons being propagated in the ejecta in the simulation, the Doppler velocity spectrum $\Phi_{\mathrm{mc}}(V)$ is deduced from Eqs. (11), (12), (16) and (17), $\forall V$ :

$$
\Phi_{\mathrm{mc}}(V)=\sum_{i=1}^{n_{\mathrm{ph}}} \sum_{k=1}^{n_{c}(i)} P_{k} \mathcal{C}_{k} \delta\left(V-V_{d, k}\right) \underset{n_{\mathrm{ph}} \rightarrow+\infty}{\longrightarrow}\langle\Phi(V)\rangle .
$$

$\langle\Phi(V)\rangle$ is the expectation value of a typical noisy PDV spectrum. As the number of photons $n_{\text {ph }}$ propagated in the MC simulation increases, the fluctuations $\sigma_{\Phi}^{2}(V)=\left\langle\Phi_{\mathrm{mc}}(V)^{2}\right\rangle-$ $\left\langle\Phi_{\mathrm{mc}}(V)\right\rangle^{2}$ observed in the Doppler spectrum are reduced.

\section{PDV Velocity Spectrograms}

Independently, PDV diagnostic is often used to get a Doppler spectrum $\Phi(V)$ on a given ejecta cloud, and experimental results may be directly compared to MC results [Eq. (11)]. A noise-corrupted spectrum is recovered, due to shot noise from the photodetector and electronic and digitization noise. For a random particle arrangement, it was shown in Ref. [10] that the probability density function of the Doppler spectrum $\Phi(V)$ measured by PDV, originating from the Gaussian fluctuations of the fields, follows a fully developed speckle statistics described by

$$
P(\Phi(V))=\frac{1}{\langle\Phi(V)\rangle+\left\langle B_{\Phi}\right\rangle} \exp \left[-\frac{\Phi(V)}{\langle\Phi(V)\rangle+\left\langle B_{\Phi}\right\rangle}\right],
$$

where $\left\langle B_{\Phi}\right\rangle$ is an additive background noise in the spectrum. The decorrelation time of intensity is directly the width of the Fourier window $T(s)$ used to process the data, and the velocity decorrelation in the spectrum is $\delta V=\lambda /(2 T)$. For a given experiment, the properties of the cloud are unknown, and it may be possible to calculate which Doppler spectrum $\langle\Phi(V)\rangle$ is the most likely to explain the observation, using Eqs. (11), (12), (17), and (18).

\section{EXAMPLE}

A single example is presented in this paper in Fig. 2. We demonstrate the relevance of performing Doppler MC simulations to illustrate particular features observed in PDV velocity spectrograms in shock compression ejecta experiments, and we show why the whole geometry of the system must be taken into account to interpret PDV data.

\section{A. Properties of the Cloud}

The areal mass-velocity distribution of ejecta $M(V)$ is supposed to be

$$
M(V)=M_{s} \exp \left[-\beta\left(\frac{V}{V_{\mathrm{fs}}}-1\right)\right] .
$$

This simple exponential model may be a good approximation in the case of small surface perturbations $[18,19]$ and for Taylor wave shocked tin [3]. $M_{s}=0.5 \mathrm{mg} / \mathrm{cm}^{2}, \beta=8.5$, $V_{\mathrm{fs}}=2000 \mathrm{~m} / \mathrm{s}$, and $V_{\max }=3600 \mathrm{~m} / \mathrm{s}$. The ejected material is tin, whose optical index at $\lambda=1.55 \mu \mathrm{m}$ is $n=$ $3.31+8.67 i[20]$ and bulk density $\rho_{p}=7.3 \times 10^{3} \mathrm{~kg} / \mathrm{m}^{3}$. The size distribution is $f_{d} \propto d_{p}^{-5.2}$ [16] with assumed minimum and maximum diameters $d_{\min }=1 \mu \mathrm{m}$ and $d_{\max }=$ $10 \mu \mathrm{m}$. Mie scattering phase functions used to propagate the photons in the medium are discretized between 0 and $\pi$ on 1000 points. For each scattering event, the particle diameter is sampled using Eq. (6). The cloud is discretized in $N=1600$ domains $\left(=V_{\max }-V_{\mathrm{fs}}\right)$ corresponding to a velocity step $1 \mathrm{~m} / \mathrm{s}$ between neighboring particle slabs. The FOV of the PDV probe (collimated beam) is $\pm \omega= \pm 250 \mu \mathrm{m}$. The initial length of the cloud is $L=1 \mathrm{~mm}$. At $t=0$, the shock releases at the metal-vacuum interface, giving rise to an expanding cloud of droplets. The polarization states of the incoming beam and of the local oscillator are linearly polarized along the

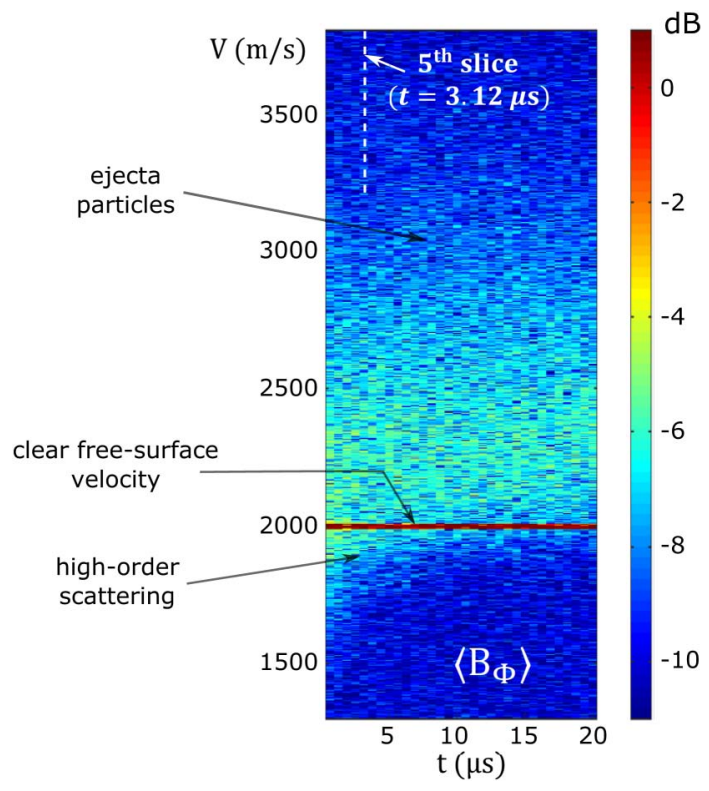

Fig. 2. Noisy Doppler velocity spectrum of an ejecta cloud expanding in vacuum, reconstructed using Eqs. (11) and (12) with $\Phi_{\mathrm{mc}} \simeq\langle\Phi(V)\rangle$. Time step $\delta t=625$ ns. $\left\langle B_{\Phi}\right\rangle=-10 \mathrm{~dB}$. 
$x$ axis (see Fig. 1): $\mathbf{S}_{0}=\left[I_{0}, Q_{0}, U_{0}, V_{0}\right]=[1,1,0,0]$. A total number of $32 \mathrm{MC}$ simulations is performed at 32 different times to reconstruct the time-velocity spectrograms near breakout. Between successive simulations, $L$ is incremented by $1 \mathrm{~mm}$ (expansion of the cloud), which corresponds to a time step $\delta t=625 \mathrm{~ns}$. The position of the PDV probe is set arbitrarily below the critical distance $d_{c}$, and simulations are performed before particles impact the probe. 15000 photons are propagated in the ejecta for each simulation (one slice of the Doppler spectrum shown in Fig. 2) and the boundaries of the cloud along $x$ and $y$ are set to $\pm 1.5 \mathrm{~mm}$, much larger than the FOV. Figure 2 shows the velocity spectrograms. At each time of motion of the particle cloud, the average spectrum $\langle\Phi(V)\rangle$ is approximated by $\Phi_{\mathrm{mc}}(V)$, determined using Doppler MC calculations. Finally, we replace $\langle\Phi(V)\rangle$ with $\Phi_{\mathrm{mc}}(V)$ in Eq. (19) to construct and represent the PDV spectrum.

\section{B. Interpretation}

A non-negligible PSD is detected below the free-surface velocity near breakout. As the cloud expands, this particular behavior progressively disappears. This feature is very likely to be explained by the multiple scattering of light in the ejecta, as also established in Ref. [10], rather than by collisions among particles (two particles impacting would potentially drag before being swept up by the surface). Our results may explain the experimental observation presented in Ref. [9] on gold ejecta production (micro-jetting) under explosively driven shock wave compression, and may be supported by the experimental observations presented in Ref. [21] on tin plate ejecta experiments: as the areal mass of ejecta increases with shock breakout pressure, the amplitude below the free-surface velocity appears in the PDV spectrum. At later times, there is as much multiple scattering in the ejecta as just after shock breakout. However, high-order scattering events occur outside the FOV (Fig. 1), and photons cannot be collected due to the very limited aperture angle of the probe $\left(\simeq 0.1^{\circ}\right)$.

Figure 3 shows the different contributions to the Doppler spectrum. As the areal mass is small $\left(0.5 \mathrm{mg} / \mathrm{cm}^{2}\right)$, a semitransparent cloud is observed. Unscattered photons can go through the cloud before being reflected by the free surface (red curve). The single scattering (SS) approximation is invalid below $V \simeq 2800 \mathrm{~m} / \mathrm{s}$, even for a very moderate areal mass. The number of scatterings depicted in Fig. 3 does not exceed 9 (for visibility), and above $\sim 15$ th order in our calculation, the contribution to the Doppler spectrum is negligible due a predominant absorption (the photon's weight approaches 0). A strong amplitude in the spectrum at $V=V_{\mathrm{fs}}$ is obtained for scattering orders between 1 and $\sim 5$. Since particle sizes $d_{p} \sim \lambda$, photons are preferentially scattered in the forward direction and, because they do not acquire any Doppler shift $\left(\mathbf{u}_{i+1}-\mathbf{u}_{i} \sim \mathbf{0}\right)$, they do not contribute to the creation of the time-velocity spectrogram. Consequently, even after few scatterings, the largest contribution to the Doppler shift accumulated by the photon may come from the reflection on the free surface. This explanation may also justify why, in many ejecta experiments, a dark region between the ejecta cloud and the free-surface velocity is observed on PDV time-velocity spectrograms [21]. Figure 4 illustrates how the geometrical properties

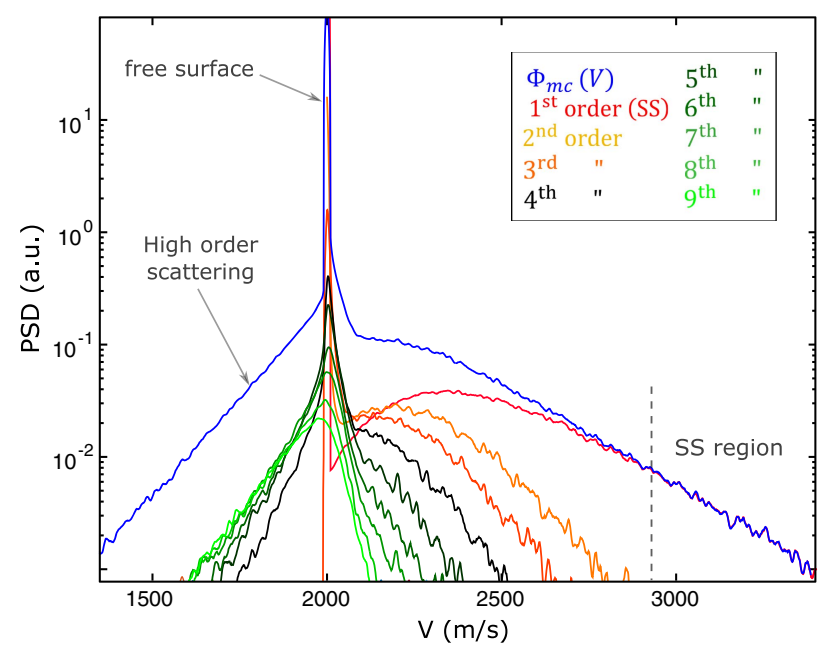

Fig. 3. Doppler velocity spectrum $\Phi_{\mathrm{mc}}$ at time $t=3.12 \mu \mathrm{s}$ (corresponding to the fifth slice shown in Fig. 2). SS is limited to the high-velocity region, and the non-zero power spectral density (PSD) detected below the free surface is justified by high-order scattering.

of the probe influence the contribution of multiple scattering to the Doppler spectrum. At a given time of motion of the cloud, this contribution increases with the beam diameter $2 \omega$, showing that the whole geometry of the system must be taken into account to interpret precisely PDV data.

Figure 5 shows the distribution of the backscattered polarization states on the surface of the Poincare sphere, after three (top) and five (bottom) scattering events undergone by the photons inside the FOV. As expected, we observe the progressive randomization of the backscattered polarizations (for an incoming linearly polarized light along the $x$ axis). As the beam goes deeper in the ejecta and the scattering order increases, the backscattered light gets progressively depolarized.

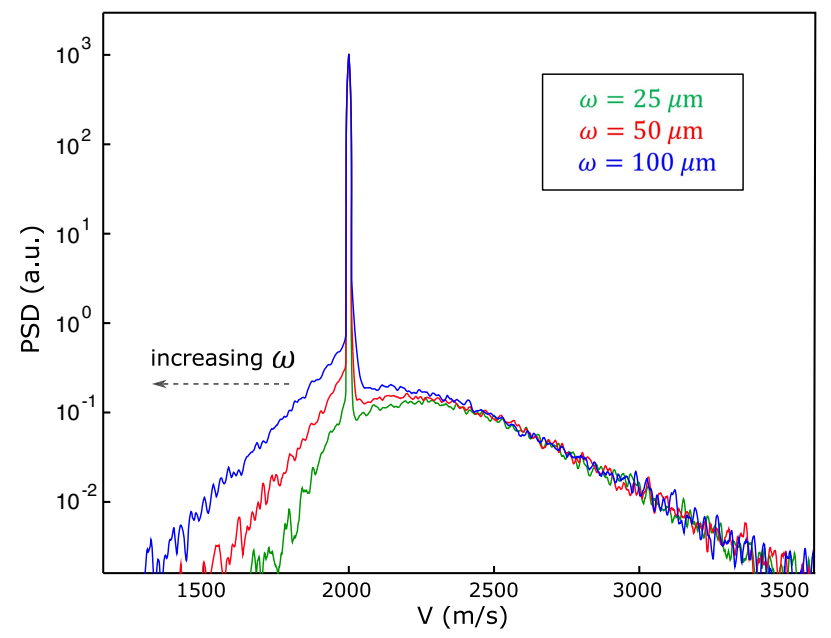

Fig. 4. Doppler velocity spectra $\Phi_{\mathrm{mc}}$ calculated for a 1 -mm-thick tin cloud (using 10000 photons) for three different beam waists $\omega$. The properties of the cloud are described in Section A. 

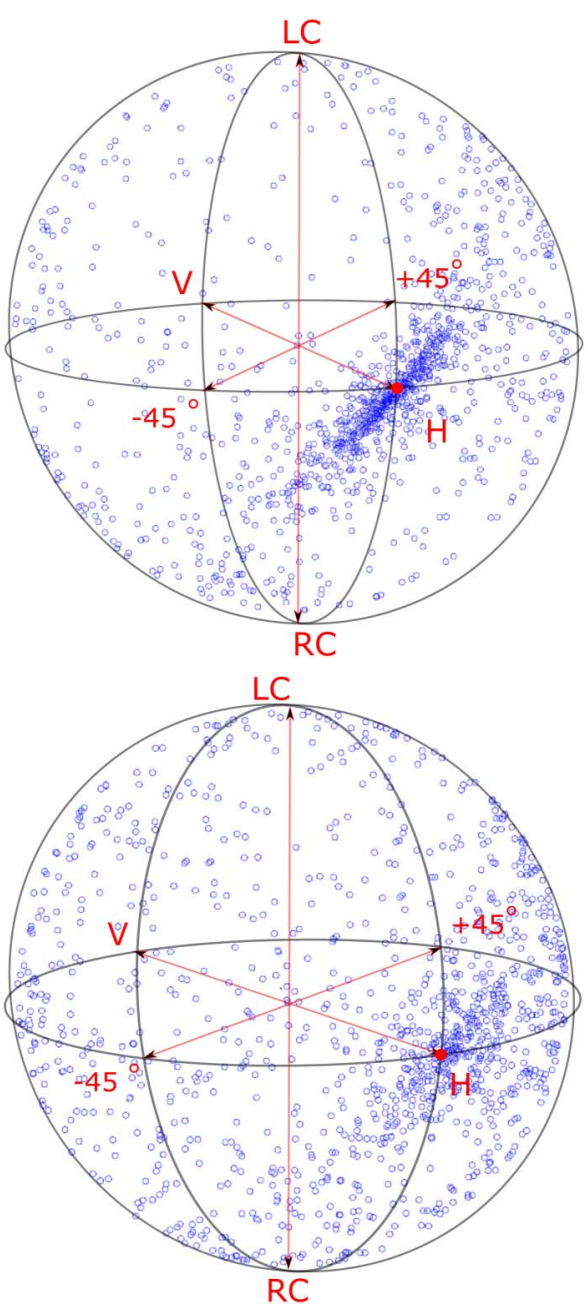

Fig. 5. Distribution of the backscattered polarization states (blue) on the surface of the Poincaré sphere after three (top) and five (bottom) scatterings undergone by the photons inside the FOV; red dot point: incoming polarization; RC: right-handed circular polarization; LC: left-handed circular polarization; $\mathrm{H}$ : horizontally polarized light (along $x$ ); V: vertically polarized light (along $y$ ); $\pm 45^{\circ}$ : linearly polarized light at $\pm 45^{\circ}$ with respect to $x$ (see Fig. 1). The properties of the cloud are defined in Section. A.

\section{DISCUSSION}

The only work dealing with multiple light scattering in metallic ejecta clouds is presented in Ref. [10]. The authors apply a twoflux Kubelka-Munk solution [22] of the radiative transfer equation (RTE) to estimate the Doppler spectrum and explain experimental PDV data. A two-flux theory supposes that the incident light is directly transformed into a diffused flux as it enters the ejecta. This may not be a realistic assumption in the case of a semi-transparent cloud, since the fastest region is very dilute, and the beam cannot be immediately diffused. Furthermore, a two-flux theory cannot be used for collimated light [22]. They use a scattering coefficient $\sigma$, which is the same for the backward and forward propagating diffused fluxes. This may suppress the strong angle dependence of scattering and induce an overestimation of the single scattering contribution to the Doppler spectrum, since the probability of backscattering is actually much smaller than the probability of forward scattering for $d_{p} \sim \lambda$. Since a clear transition between single and multiple scattering regimes appears as the beam penetrates deeper into the ejecta, MC simulations or a complete solution of the time-dependent RTE are necessary to solve the problem (the polydispersity may be introduced with difficulty in the RTE). At later times, high-order multiple scattering may have a negligible impact on the Doppler signature and may be neglected to speed up the calculation and facilitate a possible inverse approach of determining ejecta properties using noninvasive PDV diagnostic. If the areal mass of ejecta is known, MC simulations can be performed to find out which particle size distribution is the most likely to explain the data. When a larger areal mass of particles is ejected (typically 5 to $\sim 15 \mathrm{mg} / \mathrm{cm}^{2}$ ), light scattering in the ejecta may be dependent, i.e., correlations between particles in the medium must be taken into account. In this regime, the relationship between the extinction coefficient of light and the particle volume fractions is non-linear (as opposed to independent scattering). Preliminary work on the interaction between a near infrared beam and a dense distribution (up to volume fractions $20 \%)$ of micrometer-sized metallic particles shows that the second-order Keller approximation [23] to the extinction coefficient may well describe this dependent scattering regime. Such macroscopic variables may be integrated in the MC calculation to study the interaction of a PDV beam with a dense particle distribution, a situation that may be encountered when the ejecta are dragging in a gas.

\section{CONCLUSION}

In conclusion, this article addresses the multiple scattering of light in an expanding cloud. Using Doppler MC calculations incorporating the transport of polarization, we explain particular features observed in PDV Doppler spectra measured in shock compression ejecta experiments, and we show that the whole geometry of the system studied must be taken into account to interpret precisely PDV data. Extensive MC simulations are being carried out to improve our current understanding of ejecta physics probed by PDV and to reveal possibly hidden information encoded in the spectrum. For particle sizes and PDV wavelength on the same order of magnitude, multiple light scattering in the ejecta must be taken into account, even for moderate areal mass densities.

\section{REFERENCES}

1. G. Dimonte, C. Frerking, and M. Schneider, "Richtmyer-Meshkov instability in the turbulent regime," Phys. Rev. Lett. 74, 4855-4858 (1995).

2. J. Asay, W. Buttler, and J. Perry, "Ejection of material from shocked surfaces," Appl. Phys. Lett. 29, 284-287 (1976).

3. S. K. Monfared, D. M. Oró, M. Grover, J. E. Hammerberg, B. M. LaLone, C. L. Pack, M. M. Schauer, G. D. Stevens, J. B. Stone, W. D. Turley, and W. T. Buttler, "Experimental observations on the links between surface perturbation parameters and shock-induced mass ejection," J. Appl. Phys. 116, 063504 (2014).

4. M. B. Zellner, M. Grover, J. E. Hammerberg, R. S. Hixson, A. J. Iverson, G. S. Macrum, K. B. Morley, A. W. Obst, R. T. Olson, J. R. Payton, P. A. Rigg, N. Routley, G. D. Stevens, W. D. Turley, L. Veeser, and W. T. Buttler, "Effects of shock-breakout pressure 
on ejection of micron-scale material from shocked tin surfaces," J. Appl. Phys. 102, 013522 (2007).

5. M. Zellner and W. Buttler, "Exploring Richtmyer-Meshkov instability phenomena and ejecta cloud physics," Appl. Phys. Lett. 93, 114102 (2008).

6. O. T. Strand, D. Goosman, C. Martinez, T. Whitworth, and W. Kuhlow, "Compact system for high-speed velocimetry using heterodyne techniques," Rev. Sci. Instrum. 77, 083108 (2006).

7. E. A. Moro, M. E. Briggs, and L. M. Hull, "Defining parametric dependencies for the correct interpretation of speckle dynamics in photon Doppler velocimetry," Appl. Opt. 52, 8661-8669 (2013).

8. W. J. Warren, E. A. Moro, M. E. Briggs, and E. B. Flynn, "Simulating translation-induced laser speckle dynamics in photon Doppler velocimetry," Appl. Opt. 53, 4661-4668 (2014).

9. B. La Lone, G. Stevens, W. Turley, L. Veeser, and D. Holtkamp, Spall Strength and Ejecta Production of Gold Under Explosively Driven Shock Wave Compression (National Security Technologies, 2013).

10. A. V. Andriyash, M. V. Astashkin, V. K. Baranov, A. G. Golubinskii, D. A. Irinichev, A. N. Kondrat'ev, S. E. Kuratov, V. A. Mazanov, D. B. Rogozkin, S. N. Stepushkin, and V. Y. Khatunkin, "Optoheterodyne Doppler measurements of the ballistic expansion of the products of the shock wave-induced surface destruction: experiment and theory," J. Exp. Theor. Phys. 122, 970-983 (2016).

11. J. C. Ramella-Roman, S. A. Prahl, and S. L. Jacques, "Three Monte Carlo programs of polarized light transport into scattering media: part I," Opt. Express 13, 4420-4438 (2005).

12. H. Jentink, F. De Mul, R. Hermsen, R. Graaff, and J. Greve, "Monte Carlo simulations of laser Doppler blood flow measurements in tissue," Appl. Opt. 29, 2371-2381 (1990).

13. F. D. de Mul, M. Koelink, M. Kok, P. Harmsma, J. Greve, R. Graaff, and J. Aarnoudse, "Laser Doppler velocimetry and Monte Carlo simulations on models for blood perfusion in tissue," Appl. Opt. 34, 6595-6611 (1995).
14. D. S. Sorenson, P. D. Pazuchanics, R. P. Johnson, T. W. Tunnell, D. D. Smalley, R. M. Malone, M. I. Kaufman, D. G. Marks, G. A. Capelle, M. Grover, G. D. Stevens, B. M. LaLone, B. F. Marshall, and W. D. Turley, "Ejecta particle size measurements from the break-up of micro-jets in vacuum and helium gas using ultraviolet in-line Fraunhofer holography," AIP Conf. Proc. 1793, 100026 (2017).

15. A. Garca-Valenzuela, H. Contreras-Tello, J. Olivares, and F. Cuppo, "Insights into the dependent-scattering contributions to the extinction coefficient in highly scattering suspensions," J. Opt. Soc. Am. A 30, 1328-1334 (2013).

16. D. Sorenson, R. Minich, J. Romero, T. Tunnell, and R. Malone, "Ejecta particle size distributions for shock loaded Sn and Al metals," J. Appl. Phys. 92, 5830-5836 (2002).

17. J. W. Goodman, Speckle Phenomena in Optics: Theory and Applications (Roberts \& Company, 2007).

18. O. Durand and L. Soulard, "Mass-velocity and size-velocity distributions of ejecta cloud from shock-loaded tin surface using atomistic simulations," J. Appl. Phys. 117, 165903 (2015).

19. G. Dimonte, G. Terrones, F. Cherne, and P. Ramaprabhu, "Ejecta source model based on the nonlinear Richtmyer-Meshkov instability," J. Appl. Phys. 113, 024905 (2013).

20. E. Palik and G. Ghosh, Handbook of Optical Constants of Solids (Academic, 1998).

21. W. T. Buttler, D. M. Or'o, R. T. Olson, F. J. Cherne, J. E. Hammerberg, R. S. Hixson, S. K. Monfared, C. L. Pack, P. A. Rigg, J. B. Stone, and G. Terrones, "Second shock ejecta measurements with an explosively driven two-shockwave drive," J. Appl. Phys. 116, 103519 (2014).

22. A. Ishimaru, Wave Propagation and Scattering in Random Media (Academic, 1978), vol. 2.

23. A. Ishimaru and $Y$. Kuga, "Attenuation constant of a coherent field in a dense distribution of particles," J. Opt. Soc. Am 72, 13171320 (1982). 